\title{
SOME THEOREMS CONCERNING 2-DIMENSIONAL BROWNIAN MOTION
}

\author{
BY \\ FRANK SPITZER
}

This paper consists of three separate parts $\left({ }^{1}\right)$ which are related mainly in that they treat different stochastic processes which arise in the study of plane brownian motion. $\S 1$ is concerned with the process $R(t)=|Z(t)|$, denoting the distance of the 2-dimensional separable Bachelier-Wiener process $Z(t)$ $=X(t)+i Y(t)$ from the origin. We shall derive a law of the so-called strong type concerning the frequency of small values of $R(t)$. This theorem disproves a conjecture of Paul Lévy. In the next section we study the process $\theta(t)=\arg Z(t)$. Results are obtained concerning the transition probabilities and absorption probabilities of $\theta(t)$. The limiting distribution of $\left(2^{-1} \log t\right)^{-1} \theta(t)$ is found to be the Cauchy distribution. This problem has also been considered by $\mathrm{P}$. Lévy, who showed that the distribution of $\theta(t)$ must have infinite variance. The two-sided absorption time is shown to be a random variable which has a finite $n$th moment if and only if the wedge which constitutes the absorbing barrier has an interior angle $\beta<\pi / 2 n$. In $\S 3$ we point out how plane brownian motion can be used to represent the Cauchy process. A theorem on brownian motion due to $\mathrm{P}$. Lévy is then used to gain information about the Cauchy process $C(t)$. If $-1<C(0)=x<1$ the probability that $C(t) \geqq 1$ before $C(t) \leqq-1$ is found to be $1 / 2+\pi^{-1} \sin ^{-1} x$.

1. In his recent book on brownian motion [4, pp. 59-60] P. Lévy quotes a result of Dvoretzky and Erdös [3, Theorem 5] concerning brownian motion in $n \geqq 3$ dimensions. He goes on to point out that the analogue of their theorem for the plane could be found if one had an asymptotic estimate for the probability

$$
H\left(t_{1}, t_{2} ; r\right)=\operatorname{Pr}\left[\min _{t_{1} \leqq t_{2}} R(t)<r \mid R(0)=0\right], \quad \text { as } r \rightarrow 0 .
$$

We shall find such an estimate and call it

Lemia 1.

Received by the editors September 8, 1956.

(1) The results of $\$ 1$ and part of $\S 2$ are taken from the author's 1953 Ph.D. dissertation, and were presented to the Mathematical Society in Abstracts 247 and 248, December 1952. The result of $\$ 1$ was also obtained independently in 1954 by P. Erdös, A. Dvoretzky, and S. Kakutani, who kindly urged the author to publish it. 


$$
H\left(t_{1}, t_{2} ; r\right) \sim \frac{\log t_{2}-\log t_{1}}{\log r^{-2}}
$$

Using this lemma, we can prove that the correct analogue of the result of Dvoretzky and Erdös is

THEOREM 1. For every positive nonincreasing $g(t), R(t) \geqq g(t) t^{1 / 2}$ for all sufficiently large $t$ with probability one or zero, according as the series $\sum_{k=1}^{\infty}[k|\log g(k)|]^{-1}$ converges or diverges. $R(0)$ is arbitrary.

P. Lévy's conjecture that, for every $\epsilon>0, R(t)>t^{-\epsilon}$ for all sufficiently large $t$ with probability one, is therefore false. $t^{-\epsilon}$ does not decrease rapidly enough but for instance $t^{-\log t}$ does.

Lévy also points out in this context that, if $R(0)=0$, the invariance of the process $t^{-1 / 2} R(t)$ under the transformation $t \rightarrow t^{-1}$ transforms results of the type discussed into interesting local theorems. By this device, Theorem 1 produces the

Corolla RY. For positive, nonincreasing $g(t)$, and $R(0)=0, R(t) \geqq t^{1 / 2} g(1 / t)$ for all sufficiently small positive values of $t$ with probability zero or one according as the series in Theorem 1 converges or diverges.

To arrive at Lemma 1 we make the definitions

$$
\begin{aligned}
F(r, t ; R) & =\operatorname{Pr}[R(t)<r \mid R(0)=R], \\
G(r, t ; R) & =\operatorname{Pr}\left[\min _{0 \leqq \tau \leqq t} R(\tau)<r \mid R(0)=R\right] \text { if } R \geqq r, \\
& =1 \text { if } R \leqq r . \\
\hat{F}(r, \lambda ; R) & =\int_{0}^{\infty} e^{-\lambda t} F(r, t ; R) d t, \\
\hat{G}(r, \lambda ; R) & =\int_{0}^{\infty} e^{-\lambda t} G(r, t ; R) d t .
\end{aligned}
$$

It will not be necessary to compute the probabilities $F$ and $G$ explicitly. $F(r, t ; R)$ satisfies the backward diffusion equation

$$
2 \frac{\partial F}{\partial t}=\frac{\partial^{2} F}{\partial R^{2}}+\frac{1}{R} \frac{\partial F}{\partial R} .
$$

Since $F(r, t ; R) \rightarrow 0$ as $t \rightarrow 0$, it follows that

$$
\frac{\partial^{2} \hat{F}}{\partial R^{2}}+\frac{1}{R} \frac{\partial \hat{F}}{\partial R}-2 \lambda \hat{F}=0 .
$$

The general solution of (1.2) is 


$$
A I_{0}\left((2 \lambda)^{1 / 2} R\right)+B K_{0}\left((2 \lambda)^{1 / 2} R\right),
$$

where $A, B$ may depend on $\lambda$ and on $r$, and $I_{0}(z), K_{0}(z)$ are Bessel functions in standard notation. As $R \rightarrow \infty$, only $K_{0}$ is regular so that

$$
\hat{F}(r, \lambda ; R)=B(\lambda, r) K_{0}\left((2 \lambda)^{1 / 2} R\right) \text {. }
$$

Next we make use of the continuity of the sample functions and of the Markovian nature of the process $R(t)$ to write down the following renewaltype integral equation

$$
F(r, t ; R)=\int_{0}^{t} F(r, t-s ; r) d_{s} G(r, s ; R),
$$

It follows by taking the Laplace transform that for $r \leqq R$

$$
\hat{G}(r, \lambda ; R)=\frac{1}{\lambda} \frac{\hat{F}(r, \lambda ; R)}{\hat{F}(r, \lambda ; r)}=\frac{1}{\lambda} \frac{K_{0}\left((2 \lambda)^{1 / 2} R\right)}{K_{0}\left((2 \lambda)^{1 / 2} r\right)} .
$$

But, as $z \rightarrow 0, K_{0}(z)=-\log z+C \log 2+o(|z|)$, so that

$$
\lim _{r \rightarrow 0} \log \left(\frac{1}{r}\right) \hat{G}(r, \lambda ; R)=\frac{1}{\lambda} K_{0}\left((2 \lambda)^{1 / 2} ; R\right) .
$$

Now it can be verified by studying the inversion integrals of the Laplace transforms in (1.4) and (1.5), that

$$
\lim _{r \rightarrow 0} \log \left(\frac{1}{r}\right) G(r, t ; R)=\int_{R^{2} / 2 t}^{\infty} \frac{e^{-x}}{2 x} d x .
$$

Lemma 1 now follows, since

$$
H\left(t_{1}, t_{2} ; r\right)=\int_{0}^{\infty} G\left(r, t_{1}-t_{2} ; \rho\right) d F\left(\rho, t_{1} ; 0\right) .
$$

We find

$\lim _{r \rightarrow 0} \log \left(\frac{1}{r}\right) H\left(t_{1}, t_{2} ; r\right)=\frac{1}{t_{1}} \int_{0}^{\infty} \rho \exp \left(\frac{\rho^{2}}{2 t_{1}}\right) d \rho \int_{\rho^{2} / 2\left(t_{2}-t_{1}\right)}^{\infty} \frac{e^{-x}}{2 x} d x=\frac{1}{2} \log \frac{t_{2}}{t_{1}}$.

The proof of Theorem 1 follows closely that given in [2] by Dvoretzky and Erdös for the case $n \geqq 3$. There is no loss in assuming $R(0)=0$. since plane brownian motion is a recurrent process. We first assume that the series in Theorem 1 converges and define the events

$$
\begin{aligned}
A_{n} & \equiv R(t)<2^{n / 2} g\left(2^{n-1}\right) \text { for some } 2^{n-1} \leqq t \leqq 2^{n}, \quad n=1,2, \cdots, \\
A & =\limsup _{n \rightarrow \infty} A_{n} .
\end{aligned}
$$

It is seen that 


$$
\begin{aligned}
1-\operatorname{Pr}[R(t) & \left.\geqq t^{1 / 2} g(t) \text { for all sufficiently large } t\right] \\
& =\operatorname{Pr}\left[R(t)<t^{1 / 2} g(t) \text { for arbitrarily large } t\right] \leqq \operatorname{Pr}[A] .
\end{aligned}
$$

It is well known that $F(r, t ; R)$ and $G(r, t ; R)$ can be expressed as functions of the variables $t^{-1 / 2} r$ and $t^{-1 / 2} R$. Hence

$$
H\left(t_{1}, t_{2} ; r\right)=H\left(c^{2} t_{1}, c^{2} t_{2} ; c r\right), \quad c>0 .
$$

Equation (1.8) and Lemma 1 imply

$$
\operatorname{Pr}\left[A_{n}\right]=H\left(1,2 ; 2^{1 / 2} g\left(2^{n-1}\right)\right)=-\frac{\log 2}{2 \log \left[2^{1 / 2} g\left(2^{n-1}\right)\right]}\left(1+\delta_{n}\right), \quad \lim _{n \rightarrow \infty} \delta_{n}=0 .
$$

Our assumption about $g(t)$ shows that $\sum_{n-1}^{\infty} \operatorname{Pr}\left[A_{n}\right]$ converges, so that $\operatorname{Pr}[A]=0$, and by (1.7) the event of Theorem 1 is certain.

To prove the second part of Theorem 1 we assume that $g(t)$ has the property that the series diverges. Since $\log g\left(2^{n}\right)$ is a monotone function of $n$, there exists an increasing sequence $\left\{n_{k}\right\}$ of integers with the property that, as $k \rightarrow \infty$

$$
n_{k+1}-n_{k} \rightarrow \infty, \quad \sum\left|\log g\left(2^{n_{k}}\right)\right|^{-1}=\sum\left[n_{k}\left|\log g\left(n_{k}\right)\right|\right]^{-1}=\infty .
$$

Now the events $B_{k}$ and $B$ are defined as

$$
\begin{aligned}
B_{k} & \equiv R(t)<2^{n_{k} / 2} g\left(2^{n_{k}+1}\right) \text { for some } 2^{n_{k}} \leqq t \leqq 2^{n_{k}+1}, \\
B & =\limsup _{k \rightarrow \infty} B_{k},
\end{aligned}
$$

so that

$$
\operatorname{Pr}\left[R(t)<t^{1 / 2} g(t) \text { for arbitrarily large } t\right] \geqq \operatorname{Pr}[B] .
$$

By the method used above, it follows from equation (1.8) and (1.9) that

$$
\sum_{1}^{\infty} \operatorname{Pr}\left[B_{k}\right]=\infty \text {. }
$$

If the events $B_{k}$ were independent we would have $\operatorname{Pr}[B]=1$ by the BorelContelli Lemma, so that (1.10) would show that the probability in Theorem 1 is zero. We can in fact conclude that $\operatorname{Pr}[B]=1$, because the choice of the sequence $n_{k}$ has made the events $B_{k}$ almost independent. Rather than giving a detailed proof we refer to the extension of the Borel-Contelli lemma of Chung and Erdös [1], the conditions of which can be verified to hold for the sequence of events $B_{k}$.

2. Now we define the process $\theta_{R}(t)$, the angular part of the plane brownian motion. The subscript $R$ denotes the radial initial condition, i.e. $|X(0)+i Y(0)|$ $=R>0 . \theta_{R}(t)$ is then the winding number of the continuous path $Z(\tau)$ $=X(\tau)+i Y(\tau), 0 \leqq \tau \leqq t$, about the origin. Or, in other words, one may imagine the original brownian motion to take place on the Riemann surface of 
$\log z$, so that $\theta_{R}(t)=\operatorname{Im} \log [Z(t)]$. The initial condition $\theta_{R}(0)$ is left unspecified for the moment. It is clear that $\theta_{R}(t)$ will be a continuous function of $t$ with probability one (the probability is zero that $Z(t)=0$ in any $t$-interval). However a little intuition shows that $\theta_{R}(t)$, unlike $R(t)$, is not a Markov process. Nevertheless some interesting questions can be answered concerning this process. It was first considered by P. Lévy [3, p. 252] who deduced that the distribution of $\theta_{R}(t)$ must have infinite variance from the fact that $\theta_{R}(t)$ tends to assume very large values, when $R(t)$ is small. We return to this problem after studying the absorption problem which, in a way, is more general.

Let $\theta_{R}(0)=\alpha>0$, and $\beta>\alpha$, and let

$$
T=T_{R, \alpha, \beta}=\inf _{\tau}\left[\tau \mid \theta_{R}(\tau) \leqq 0, \text { or } \theta_{R}(\tau) \geqq \beta\right] .
$$

Then $u(R, \alpha, t)=\operatorname{Pr}[T>t]=\operatorname{Pr}\left[0<\theta_{R}(\tau)<\beta\right.$, for all $\left.0 \leqq \tau \leqq t\right]$. This absorption distribution satisfies the diffusion equation in polar coordinates as its backward equation, and since the process $Z(t)$ is recurrent, it is determined by the boundary and initial value problem

$$
\begin{gathered}
2 \frac{\partial u}{\partial t}=\frac{\partial^{2} u}{\partial R^{2}}+\frac{1}{R} \frac{\partial u}{\partial R}+\frac{1}{R^{2}} \frac{\partial^{2} u}{\partial \alpha^{2}}, \quad R>0, t>0,0<\alpha<\beta, \\
u(R, \alpha, 0)=1, \quad u(R, 0, t)=u(R, \beta, t)=0 .
\end{gathered}
$$

This problem has been solved by many authors, but we prefer to use a new method which gives the solution in an elegant form. Let

$$
v(s, \alpha, t)=\left(\frac{2}{\pi s}\right)^{1 / 2} \int_{0}^{\infty} u(r, \alpha, t) e^{-r^{2} / 2 s} d r,
$$

One verifies, through integration by parts, that $v$ is the solution of the problem

$$
\begin{gathered}
\frac{\partial^{2} v}{\partial \alpha^{2}}+4 s^{2} \frac{\partial^{2} v}{\partial s^{2}}+4 s \frac{\partial v}{\partial s}-4 s^{2} \frac{\partial^{2} v}{\partial s \partial t}-2 s \frac{\partial v}{\partial t}=0, \\
v(s, \alpha, 0)=1, \quad v(s, 0, t)=v(s, \beta, t)=0 .
\end{gathered}
$$

It remains to use the invariance of $u$ under transformations of the state space leaving $R t^{-1 / 2}$ invariant, as was done for the function $H\left(t_{1}, t_{2} ; r\right)$ in the last section. Hence $u$ is a function of $\alpha$ and $R t^{-1 / 2}$, and $v$ is a function of $\alpha$ and $t s^{-1}$. Now let

$$
t^{1 / 2}=s^{1 / 2} \sinh y, \quad v(s, \alpha, t)=w(\alpha, y) .
$$

By this change of variable (2.3) now reduces to the simple Dirichlet problem

$$
\begin{gathered}
\frac{\partial^{2} w}{\partial \alpha^{2}}+\frac{\partial^{2} w}{\partial y^{2}}=0, \quad y>0,0<\alpha<\beta, \\
w(\alpha, 0)=1, \quad w(0, y)=w(\beta, 0)=0 .
\end{gathered}
$$


The solution is

$$
w(\alpha, y)=\frac{2}{\pi} \tan ^{-1}\left[\sin \frac{\alpha \pi}{\beta} / \sinh \frac{y \pi}{\beta}\right],
$$

and the distribution of $T_{R, \alpha, \beta}$ can be obtained through inversion of the integral transform in (2.2).

If $\beta=\pi$, then it is known that $T_{r, \alpha, \pi}$ is a positive stable random variable of index one-half, since it is the time before absorption in a half-plane. Therefore it has an infinite first moment. On the other hand, the time of absorption in a strip has also been studied. It is the same random variable as the absorption time for an interval in one-dimensional brownian motion and has moments of all orders. (This distribution is contained as a special case in the present model, from which it can be obtained by letting $\beta \rightarrow 0$, and $R \rightarrow \infty$ so that $R \sin \beta$ =const.) The time to absorption in a wedge is now found to have the interesting behavior that the existence of moments depends on the angle of the wedge.

THEOREM 2. For $\delta \geqq 0,\left(T_{R, \alpha, \beta}\right)^{\delta}$ has finite expected value if and only if $2 \delta \beta<\pi$. This criterion is independent of the initial position $R>0$ and of $\alpha$ with $0<\alpha<\beta$.

Proof. In the terminology of Markov processes all states inside the wedge communicate. Hence the moment of order $\delta$ is either finite or infinite, independent of $R$ and of $\alpha$. For simplicity we therefore set $\alpha=\beta / 2$, and equations (2.2) and (2.4) imply that

$$
E\left[T_{R, \beta / 2, \beta}^{\delta}\right]=-\int_{0}^{\infty} t_{d}^{\delta} u\left(R, \frac{\beta}{2}, t\right)
$$

is finite or infinite according as the integral

$$
-\int_{0}^{\infty} t^{\delta} d_{t} w\left[\frac{\beta}{2}, \sinh ^{-1}\left(\frac{t}{s}\right)^{1 / 2}\right]
$$

converges or diverges. Making use of (2.6), the criterion of Theorem 2 is readily verified.

Next we let $\beta \rightarrow \infty$ to obtain one-sided absorption probabilities. Because of the evident symmetry of the process $\theta_{R}(t)$ about $\theta_{R}(0)=0$, we can write

$$
\begin{aligned}
u_{0}(R, \alpha, t) & =\lim _{\beta \rightarrow \infty} u(R, \alpha, t)=\operatorname{Pr}\left[\min _{0 \leqq \tau \leqq t} \theta_{l R}(\tau)>0 \mid \theta_{R}(0)=\alpha\right] \\
& =\operatorname{Pr}\left[\max _{\theta \leqq \tau \leqq t} \theta_{l i}(\tau)<\alpha \mid \theta_{l i}(0)=0\right] .
\end{aligned}
$$

Since 


$$
\lim _{\beta \rightarrow \infty} \omega(\alpha, y)=\frac{2}{\pi} \tan ^{-1} \frac{\alpha}{y},
$$

we have from (2.2) and (2.4)

$$
\left(\frac{2}{\pi s}\right)^{1 / 2} \int_{0}^{\infty} u_{0}(r, \alpha, t) e^{-r^{2} / 2 s} d r=\frac{2}{\pi} \tan ^{-1} \frac{\alpha}{\log \left[(1+t / s)^{1 / 2}+(t / s)^{1 / 2}\right]}
$$

Before inverting the integral transform in (2.8) we prove a lemma which shows how to obtain the transition probabilities of the process $\theta_{R}(t)$.

Lemma 2. The transition probability $P_{R}(\alpha, t)=\operatorname{Pr}\left[\theta_{R}(t)<\alpha \mid \theta_{R}(0)=0\right]$ $=1 / 2+u_{0}(R, \alpha, t) / 2$, for $\alpha \geqq 0$. For $\alpha \leqq 0$ it is defined by the requirement that $\theta_{R}(t)$ be a symmetric random variable.

Proof. We make use of the continuity of the sample functions $\theta_{R}(t)$ as well as the above mentioned symmetry of $\theta_{R}(t)$. The argument used is nothing but the well known reflection principle, and it is interesting to note that it goes through for a process which is not Markovian.

Let $A$ be the event that $\max _{0 \leq \tau \leq t} \theta_{R}(\tau)>\alpha$, and $B$ the event that $\theta_{R}(t)>\alpha$, both subject to the initial condition $\theta_{R}(0)=0 . \Omega$ is the space of all sample functions. Symmetry and continuity together imply $\operatorname{Pr}[B \mid A]=\operatorname{Pr}[\Omega-B \mid A]$. Hence $\operatorname{Pr}[B \cap A]=\operatorname{Pr}[(\Omega-B) \cap A]$. But $B \subset A$, so that $\operatorname{Pr}[B]=\operatorname{Pr}[A-B]$ $=\operatorname{Pr}[A-B]$, or $\operatorname{Pr}[\Omega-B]=1 / 2+\operatorname{Pr}[\Omega-A] / 2$. But that is the content of the lemma.

From the above Lemma and from equation (2.8) we obtain

$$
\left(\frac{2}{\pi s}\right)^{1 / 2} \int_{0}^{\infty} P_{R}(\alpha, t) e^{-R^{2} / 2 s} d R=\frac{1}{2}+\frac{1}{\pi} \tan ^{-1}\left[\frac{\alpha}{\log \left((1+t / s)^{1 / 2}+(t / s)^{1 / 2}\right)}\right] .
$$

If the characteristic function of $\theta_{R}(t)$ is

$$
\phi(\lambda, t ; R)=E\left[e^{i \lambda \theta_{R}(t)} \mid \theta_{R}(0)=0\right]=\int_{-\infty}^{\infty} e^{i \lambda \alpha} d P_{R}(\alpha, t),
$$

it follows that

$$
\int_{0}^{\infty} \frac{\phi\left(\lambda, t ;(2 x)^{1 / 2}\right)}{(2 x)^{1 / 2}} e^{-p x} d x=\left(\frac{\pi}{2 p}\right)^{1 / 2}\left[(t p+1)^{1 / 2}-t^{1 / 2} p^{1 / 2}\right]^{|\lambda|} .
$$

It is not difficult to verify that the (unique) inversion of the Laplace transform in (2.9) gives

$$
\phi(\lambda, t ; R)=\left(\frac{\pi}{2}\right)^{1 / 2}\left(\frac{R^{2}}{4 t}\right)^{1 / 2} e^{-R^{2} / 4 t}\left[I_{(|\lambda|-1) / 2}\left(\frac{R^{2}}{4 t}\right)+I_{(|\lambda|+1) / 2}\left(\frac{R^{2}}{4 t}\right)\right],
$$

where $I_{\nu}(z)$ is the modified Bessel function of the first kind of order. 
Finally, (2.10) enables us to find the normalized limiting distribution of $\theta_{R}(t)$. Let

$$
\begin{array}{ccc}
\delta & =R(8 t)^{-1 / 2}, \quad \mu=-\frac{\lambda}{\log \delta}, \quad \lambda>0 . \\
\lim _{t \rightarrow \infty} \phi\left(\frac{2 \lambda}{\log \left(8 t / R^{2}\right)}, t ; R\right) & \left.=\lim _{\delta \rightarrow 0} \pi^{1 / 2} \delta e^{-2 \delta^{2}\left[I_{(\mu-1) / 2}\left(2 \delta^{2}\right)\right.}+I_{(\mu+1) / 2}\left(2 \delta^{2}\right)\right] & \\
=\lim _{\delta \rightarrow 0} \pi^{1 / 2} \delta e^{-2 \delta^{2}} \sum_{n=0}^{\infty}\left[\frac{\delta^{4 n+\mu-1}}{n ! \Gamma(n+\mu / 2+1 / 2)}+\frac{\delta^{4 n+\mu+1}}{n ! \Gamma(n+\mu / 2+3 / 2)}\right] .
\end{array}
$$

Only the first term of the first series contributes to the limit, which becomes

$$
\lim _{\delta \rightarrow 0} \frac{\pi^{1 / 2} e^{-2 \delta^{2} \delta^{\mu}}}{\Gamma(1 / 2+\mu / 2)}=e^{-\lambda}=\lim _{t \rightarrow \infty} \phi\left(\frac{2 \lambda}{\log t}, t ; R\right) .
$$

Due to the continuity theorem for characteristic functions, we have indeed proved

THEOREM 3. If $\theta_{R}(0)=0$, then the characteristic function of $\theta_{R}(t)$ is given by equation (2.10), and

$$
\lim _{t \rightarrow \infty} \operatorname{Pr}\left[\theta_{R}(t)<\frac{\alpha}{2} \log t\right]=\frac{1}{\pi} \int_{-\infty}^{\alpha} \frac{d x}{1+x^{2}} .
$$

The limit theorem could have been obtained with little effort directly from Equation (2.8) and from Lemma 2, or alternatively, directly from Equation (2.9).

3. The Cauchy process $C(t)$ is defined as the separable process with stationary independent increments, whose distribution is given by

$$
\operatorname{Pr}[C(t+s)-C(s)<x]=\frac{t}{\pi} \int_{-\infty}^{x} \frac{d \xi}{t^{2}+\xi^{2}} .
$$

This process has a very simple representation in terms of our 2-dimensional brownian motion $X(t)+i Y(t)$. We assume throughout that $X(0)=x_{0}$, $Y(0)=0$, and define for $y \geqq 0$ the random variable

$$
T(y)=\min _{0 \leqq t}[t \mid Y(t) \geqq y] .
$$

We shall leave aside the measure theoretical justification which should accompany each such definition. (Since $Y(t)$ is separable, a proof that $T(y)$ is a random variable is obtained by representing the set $[T(y)<\tau]$ as a countable union of simpler measurable subsets of the space of sample functions $Z(t)$.)

Now the Cauchy process is represented by $Z(t)$, i.e., the sets $\left[C\left(y_{k}\right) \leqq a_{k}\right.$, $k=1, \cdots, m]$ are in the Borel field of sets generated by $\left[X\left(t_{k}\right) \leqq \alpha_{k}, Y\left(t_{k}\right)\right.$ $\left.\leqq \beta_{k}, k=1, \cdots, m\right]$, by virtue of 
LEMma 3.

$$
C(y)=X[T(y)] \text {, if } C(0)=Z(0) .
$$

Proof. $X[T(y)]$ is the $x$-coordinate of $Z(t)$ at the first time when $\operatorname{Im} Z(t)$ $=y$. Hence the fact that increments $X[T(y+\eta)]-X[T(\eta)]$ are independent and stationary (their distribution is independent of $\eta$ ). Equation (3.1) follows from

$$
\operatorname{Pr}[T(y) \leqq s]=2 \operatorname{Pr}[Y(s)>t]=\left(\frac{2}{\pi}\right)^{1 / 2} \int_{y / 8^{1 / 2}}^{\infty} e^{-x^{2} / 2} d x
$$

Hence

$$
\begin{aligned}
\operatorname{Pr}[X(T(y)) \leqq x] & =\int_{0}^{\infty} \operatorname{Pr}\left[X(s) \leqq x \mid X(0)=x_{0}\right] d s \operatorname{Pr}[T(y) \leqq s] \\
& =\frac{y}{\pi} \int_{-\infty}^{x-x_{0}} \frac{y}{\xi^{2}+y^{2}} d \xi .
\end{aligned}
$$

The separability of $C(y)$ follows from that of $Z(t)$.

To exhibit the advantage of this representation we pose the simple but not trivial problem of computing the probability $P\left(x_{0}\right)$ that the Cauchy process $C(t)$, with $-1<C(0)=x_{0}<1$ enters the region $x \geqq 1$ before entering $x \leqq-1$. That the corresponding problem for 1 -dimensional brownian motion has the solution $\left(1+x_{0}\right) / 2$ is an immediate consequence of the symmetry and continuity of the process. The answer to the present problem should be different due to the discontinuities of the sample functions $C(t)$.

We define a new process

$$
M(t)=\max _{0 \leqq \tau \leqq t} Y(\tau), \quad Y(0)=0 .
$$

Now we have a picture, albeit not a simple one, of $P\left(x_{0}\right)$ as the probability of a certain set of paths of $Z(t)$ with $Z(0)=x_{0} \cdot P\left(x_{0}\right)$ is the probability that, if we observe $X(t)$ only at the instants $\tau$ when $M(\tau)-Y(\tau)=0$, the event $X(\tau) \geqq 1$ should occur before the event $X(\tau) \leqq-1$. This picture is rendered simple by a theorem of P. Lévy [3, Theorem 49.1]:

"If $Y(0)=0$, then $M(t)-Y(t)$ is the same stochastic process as $|Y(t)|$. "

A proof based on Doob's theory of separability would be similar to that of Lemma 3. It is necessary to show that each process is Markovian, that both processes have the same one-step transition probabilities, and that both are separable.

Since $X(t)$ and $Y(t)$ are independent processes, we can replace $M(t)-Y(t)$ by $|Y(t)|$ in the above description of $P\left(x_{0}\right)$. Let $E_{2}$ be the complex plane, $A$ and $B$ its subsets 


$$
A=[x \geqq 1 ; y=0], \quad B=[x \leqq-1 ; y=0] .
$$

Let

$$
u(x, y)=\operatorname{Pr}[Z(t) \in A \text { before } Z(t) \in B \mid Z(0)=x+i y],
$$

with $x+i y \in E_{2}-A \cup B$. Then $P\left(x_{0}\right)=u\left(x_{0}, 0\right)$. But it is well known that $u(x, y)$ is the solution of the Dirichlet problem

$$
\begin{gathered}
\frac{\partial^{2} u}{\partial x^{2}}+\frac{\partial^{2} u}{\partial y^{2}}=0, \quad x+i y \in E_{2}-A \cup B, \\
u(x, y)=1 \text { for } x+i y \in A \\
u(x, y)=0 \text { for } x+i y \in B .
\end{gathered}
$$

The solution is

$$
\begin{aligned}
& u(x, y)=\frac{1}{\pi} \tan ^{-1} \frac{2 \operatorname{Im}(w)}{w \bar{w}-1}, \\
& x+i y=\frac{1+w^{2}}{1-w^{2}}, \quad \operatorname{Im} w>0,
\end{aligned}
$$

so that

$$
P(x)=\frac{2}{\pi} \tan ^{-1}\left(\frac{1+x}{1-x}\right)^{1 / 2}=\frac{1}{2}+\frac{1}{\pi} \sin ^{-1} x .
$$

This is

TheOREM 4. The Cauchy process starting at $x \in(-1,1)$ is absorbed on the right with probability $1 / 2+\sin ^{-1} x / \pi$.

It is possible to do more. By changing the boundary condition in (3.2) to $u(x, y)=1$ for $x+i y$ in a measurable subset $S$ of $A \cup B$, and $u(x, y)=0$ for $x+i y$ in $A \cup B-S$, one can deduce that a Cauchy process $C(t)$ with $-1<C(0)$ $=x_{0}<1$, will first be absorbed in $A \cup B$ at a point $y$ with the probability density

$$
f\left(x_{0} ; y\right)=\frac{1}{\pi}\left(1-x_{0}^{2}\right)^{1 / 2} \cdot \frac{1}{\left|y-x_{0}\right|\left(y^{2}-1\right)^{1 / 2}}, \quad|y|>1 .
$$

Both $f(x ; y)$ and $P(x)$ satisfy the equation

$$
\text { P.V. } \int_{-1}^{1} \frac{\partial f(x ; y) / \partial x}{x-t} d x=\text { P.V. } \int_{-1}^{1} \frac{P^{\prime}(x)}{x-t} d x=0, \quad|t|<1 .
$$

It is difficult to derive these equations directly, and to impose appropriate 
conditions. $P(x)$ for instance, as given by (3.3), happens to be the unique solution of (3.4) with $P(1)=1, P(-1)=0$, and $P^{\prime}(x) \cdot\left(1-x^{2}\right)^{1 / 2}$ in $L_{2}(-1,1)$.

\section{REFERENCES}

1. K. L. Chung and P. Erdös, On the application of the Borel-Contelli lemma, Trans. Amer. Math. Soc. vol. 72 (1952) pp. 179-186.

2. A. Dvoretzky and P. Erdös, Some problems on random walk in space, Proceedings of the 2nd Berkeley Symposium, Univ. of California Press, 1951, pp. 353-368.

3. P. Lévy, Processus stochastiques et mouvement brownien, Paris, 1948.

4. ——, Le mouvement browien, Mémorial des Sciences Mathématiques, Fasc. 76, Paris, 1954.

California Institute of Technology, Pasadena 4, Calif. 